\title{
Ist eine Gewichtszunahme kardiovaskulär ungünstig?
}

\author{
Nach einem Rauchstopp nimmt das kardiovaskuläre Risiko \\ messbar ab. Negative Auswirkung des Rauchverzichts ist \\ allerdings eine Gewichtszunahme. Da auch Übergewicht ein \\ kardiovaskulärer Risikofaktor ist, könnte diese Zunahme die \\ Vorteile des Nikotinverzichts aufwiegen. \\ JAMA 2013; 309: 1014-1021
}

C. Clair et al. haben die Daten aus der Framingham Offspring Study analysiert, in der Daten von 1984-2011 erhoben wurden. Bei jeder 4-Jahres-Untersuchung wurden die Teilnehmer kategorisiert in Raucher und Nicht-Raucher. Die NichtRaucher wurden unterteilt in Nicht-Raucher, die 4 oder weniger Jahre (kürzlich) oder mehr als 4 Jahre (längerfristig) nicht mehr geraucht hatten oder die nie geraucht hatten. Endpunkte waren kardio- vaskuläre Ereignisse nach 6 Jahren sowie die Gewichtszunahme nach 4 Jahren und ihr Einfluss auf die kardiovaskulären Ereignisse. Als kardiovaskuläre Ereignisse zählten koronare und zerebrovaskuläre Ereignisse, periphere arterielle Verschlusskrankheit sowie Herzinsuffizienz.3251 Teilnehmer wurden im Mittel 25 Jahre beobachtet. In dieser Zeit kam es zu 631 kardiovaskulären Ereignissen. Die Gewichtszunahme war bei kürzlichen Ex-
Rauchern (2,7 kg bei Patienten ohne Diabetes bzw. 3,6 kg mit Diabetes) deutlich höher als bei längerfristigen Ex-Rauchern (0,9 kg bzw. 0,0 kg). Unter Nicht-Diabetikern traten bei den Rauchern 5,9 Ereignisse pro 100 Personen-Untersuchungen auf. Bei den kürzlichen Ex-Rauchern lag diese Zahl bei 3,2, bei den längerfristigen ExRauchern bei 3,1 und bei den echten Nicht-Rauchern bei 2,4. Damit sank das kardiovaskuläre Risiko bei einem Rauchstopp um $43 \%$ ( $\leq 4$ Jahre) bzw. 44\% (>4 Jahre). Dieser Zusammenhang veränderte sich nur minimal nach einer Anpassung um Gewichtsänderungen. Unter den Teilnehmern mit Diabetes ergaben sich ähnliche Schätzwerte, die jedoch statistisch nicht signifikant waren.

\section{Fazit}

In dieser Studie war ein Rauchstopp mit einem geringeren Risiko für kardiovaskuläre Ereignisse bei Nicht-Diabetikern assoziiert. Eine Gewichtszunahme beeinflusste diesen Zusammenhang nicht, so die Autoren.

\section{Dr. Christoph Feldmann, Köln}

dem persönlichen Kennenlernen, dem Gedankenaustausch mit Kollegen und Freunden, der Knüpfung neuer klinischer und wissenschaftlicher Kontakte. Dies war zum Beispiel in den Pausen mit dem Besuch der Industrieausstellung möglich sowie am Gesellschaftsabend im Prunksaal des Maharadschas im Zoo Hannover - wir fühlten uns in eine fremde, geheimnisvolle Welt versetzt. Bei der Eröffnungsfeier hielt Gerhard Thiele, Astronaut der ESA, den ausgezeichneten Festvortrag „Raumfahrt - ein anderes Bild der Erde." Wir konnten eindrucksvoll erkennen, wie dünn die Schicht der blauen Atmosphäre, in welcher wir atmen und leben können, in Wirklichkeit ist.

Zum Schluss noch einmal einen herzlichen Dank an alle Vortragenden und Vorsitzenden, die zum Gelingen des Kongresses beigetragen haben. Nach über einjähriger Vorbereitung habe ich selbst den Kongress sehr genossen. Meinen Nachfolgern, Dieter Ukena aus Bremen und Jürgen Seidenberg aus Oldenburg, wünsche ich alles Gute, viel Erfolg und Freude bei der Vorbereitung ihres Kongresses in Bremen vom 26. bis 29. März 2014.

Ulrich Costabel, Essen

DGP-Kongresspräsident 2013 